\title{
The Magneto-Resistive Magnetometer of BCU on the Tatiana-2 Satellite
}

\author{
Li-Yeh Liu ${ }^{1}$, Shyh-Biau Jiang ${ }^{1,2, *}$, Tse-Liang Yeh ${ }^{1,2}$, Huey-Ching Yeh ${ }^{3}, J^{2}$ nn-Yeng Liu ${ }^{3}$, \\ Ying-Hao $\mathrm{Hsu}^{2}$, and Ji-Yi Peng ${ }^{2}$ \\ ${ }^{1}$ Institute of Mechanical Engineering, National Central University, Jhongli, Taiwan, ROC \\ ${ }^{2}$ Institute of Opto-Mechatronics Engineering, National Central University, Jhongli, Taiwan, ROC \\ ${ }^{3}$ Institute of Space Science, National Central University, Jhongli, Taiwan, ROC
}

Received 8 August 2011, accepted 7 November 2011

\begin{abstract}
The magneto-resistive magnetometer (MRM) of the Block of Central University (BCU) payload onboard the Tatiana-2 satellite is made of anisotropic magneto-resistive (AMR) sensor chips, which have appealing features of small size $(10 \times 15$ $\left.\times 7 \mathrm{~mm}^{3}\right)$, light weight $(2 \mathrm{grams})$ and low power consumption $(100 \mathrm{~mW})$. The small MRM is packaged together with other instrument/subsystems of the BCU into a $1.6 \mathrm{~kg}$ payload box for convenient installation. In this report, we present the design, calibration, and flight data analysis of the MRM. In particular, the detailed methods of pre-flight calibrations are described. The calibrated data revealed typical patterns of the global geo-magnetic field structure and of field-aligned current (FAC) distribution in the high latitude ionosphere, though the MRM of BCU only has a resolution of $24 \mathrm{nT}$ and a sampling rate of $2.22 \mathrm{~Hz}$. Moreover, the current density derived from our magnetic field measurements are about 2 and $3 \mu \mathrm{A} \mathrm{m}^{-2}$, respectively, for downward and upward FAC, which are comparable to those typically observed at auroral latitudes during a quiet geomagnetic condition.
\end{abstract}

Key words: Tatiana-2, Ionosphere, Magneto-resistive magnetometer, AMR sensor, Pre-flight calibration, Field-aligned current, Taiwan Citation: Liu, L. Y., S. B. Jiang, T.L.Yeh, H. C. Yeh, J.Y.Liu, Y. H. Hsu, and J.Y.Peng, 2012: The magneto-resistive magnetometer of BCU on the Tatiana-2 satellite. Terr. Atmos. Ocean. Sci., 23, 317-326, doi: 10.3319/TAO.2011.11.07.01(AA)

\section{INTRODUCTION}

The purpose of the BCU payload carried by Tatiana-2 is to study space weather features as well as earthquake induced signatures in the terrestrial ionosphere. Since the Tatiana-2 micro-satellite (also known as Universitetsky-2) was to fly along a pre-noon to pre-midnight sunsynchronous circular orbit at an altitude of $\sim 830 \mathrm{~km}$ and an inclination angle of $98.8^{\circ}$, a magnetometer is an ideal choice to detect magnetic field variations associated with prominent fieldaligned current phenomenon at high latitudes.

Because of the advantage of small size and light weight, the anisotropic magneto-resistive (AMR) sensor based magnetometer have been investigated for years by the faculties and students at the Insititute of Opto-Mechatronics Engineering, National Central University (Yang 1997, 2002; Liu 2005). The MRM has an upgraded design surpassing that of the AMR magnetometer for sounding rocket experi-

\footnotetext{
* Corresponding author

E-mail: sbjiang@viewmove.com
}

ments (Chou 2008). The magnetometer is integrated with the electron temperature probe (ETP) and supporting electronic packages into the Block of Central University to form a payload for the purpose of investigating ionospheric phenomena.

In this paper we concentrate on the design, calibration and flight data analysis of the MRM. The paper is organized as follows. In section 2, we introduce the functions of the MRM. Section 3 describes the pre-flight calibration of the MRM, and section 4 shows the result of in-flight data analysis, including the findings with regard to magnetic field changes induced by field-aligned currents. The last section concludes the discussion.

\section{FUNCTIONAL DESCRIPTION}

The MRM magnetometer was built to meet the scientific requirement of the payload project off the shelf anisotropic magneto-resistive (AMR) sensors. To describe the 
implementation of the MRM's instrumental amplifying circuit, the basic characteristics and terminologies of the AMR sensor and the control timing of sensor operation will also be introduced briefly.

\subsection{The AMR Sensor}

There are many small regions called "magnetic domains" inside an AMR resistor; four AMR resistors form an AMR sensor chip in general, as shown in Fig. 1. An AMR resistor must be placed under a magnetic field strong enough to magnetize all its magnetic domains in one direction (called "easy-axis," as indicated in Fig. 1a) before it can be used to sense weak magnetic fields. In order to maintain sensitivity and error-free operation, the AMR sensor needs to be periodically magnetized by the built-in coil generated strong magnetic field in the scenario of use (Caruso et al. 1999). The measurement of three-dimensional magnetic field could also be achieved by combining three AMR sensors into a magneto-resistive vector magnetometer.

Since the AMR sensor chip is a resistive electronic component, it can be driven by constant voltage or constant current methods without complex driving circuits. Compared with fluxgate magnetometers, the magnetoresistive magnetometers have advantagous features in their small size, low power consumption and simple driving circuit.

\subsection{Functional Block Diagram}

As shown in Fig. 2, magnetic field first forces the AMR sensor to change its resistance, which is transduced into a differential voltage signal. Then the voltage signal is amplified and the offset voltage is removed. Finally the analogue voltage signals are acquired as digital data by analogto-digital converter (ADC). The offset voltage arises from several sources. The offset voltage comes from the crossaxis field contribution called the "cross-axis effect;" another offset voltage comes from an unbalanced Wheatstone bridge of the AMR sensor under a zero magnetic field circumstance called a "bridge offset." A combination of other offsets originating from the signal amplifier, offset control and ADC is called a "circuit offset."

The offset voltage will seriously degrade the ADC's dynamic range after being amplified, so we must cancel it in two ways. The payload processor can determine how much offset control voltage to be generated to cancel the dominant part of offset voltage and immediately optimize the dynamic range of signal. Any residual offset voltage is corrected by using a special mechanism called "Set/Reset" (Pant and Caruso 1996). The magnetizations of sensor are defined as "Set-state" and "Reset-state" when the sensor has been magnetized along and against the easy-axis direction, respectively. Because a reversal of the magnetizing field polarity will not affect the offset voltages resulting from bridge offset, cross axis effect and circuit offset in the sensor output, we therefore can change the magnetizing of sensors between the Set-state and Reset-state alternatively then subtract the measurements obtained in both states to eliminate any residual offset.

\subsection{Control Sequence}

In order to maintain high sensitivity and compensate the offset of the sensor, the magnetizing procedure is carried out before taking every MRM measurement (Caruso et al. 1998). For purposes of cancelling the bridge offset as well as eliminating the cross-axis effect and circuit offset, the polarity of magnetizing current is alternated between a "Set-state" and "Reset-state." As shown in Fig. 3, one full cycle of measurement contains "Set-state" switching and (a)
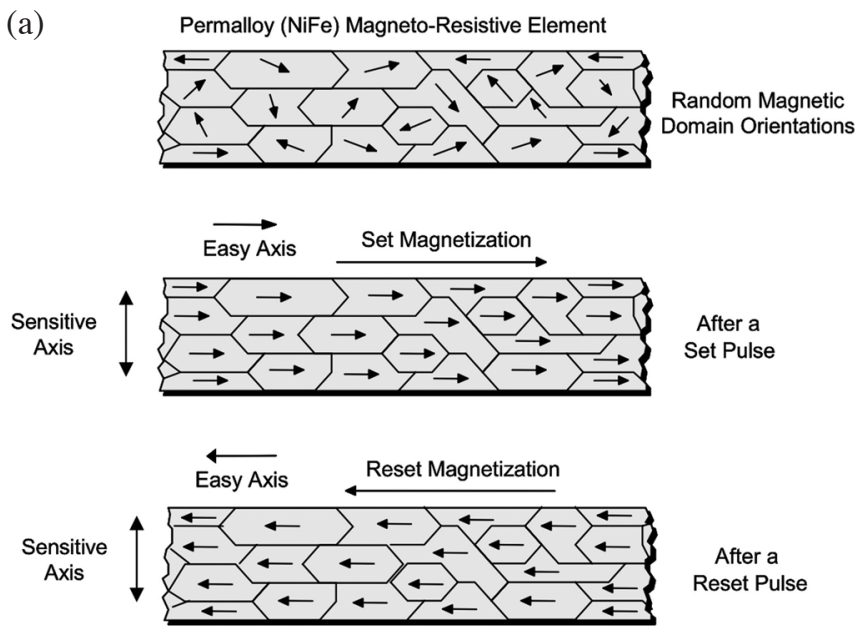

(b)

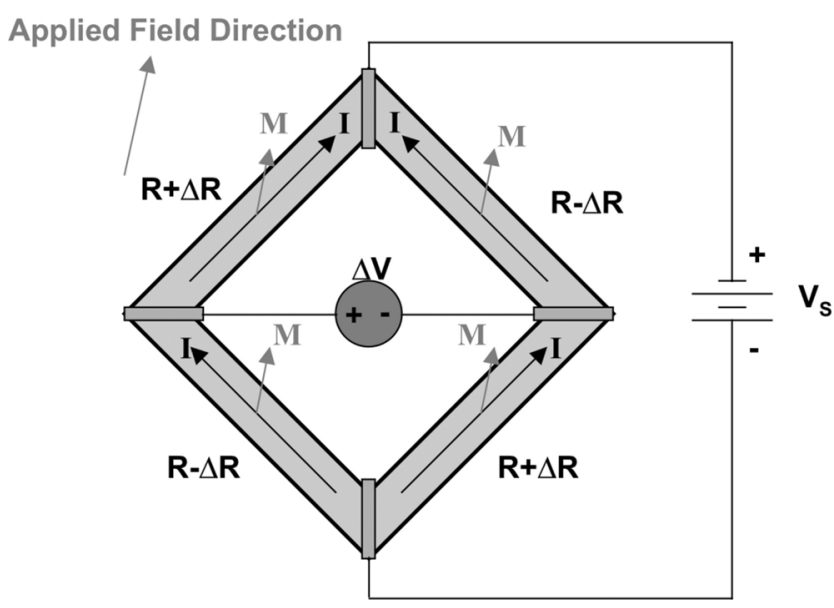

Fig. 1. A diagram of the operation principles of the AMR (Courtesy of Honeywell International, Inc.): (a) magnetic domains inside an AMR resistor with random orientation and two states of magnetization, (b) an AMR sensor consists of 4 AMR resistors. 


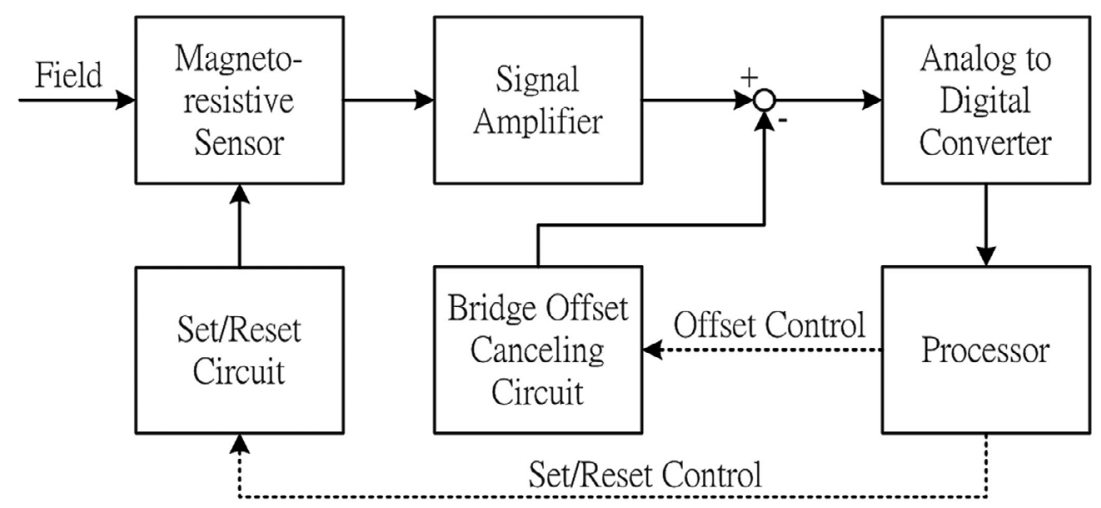

Fig. 2. Functional block diagram (only one axis is shown here).

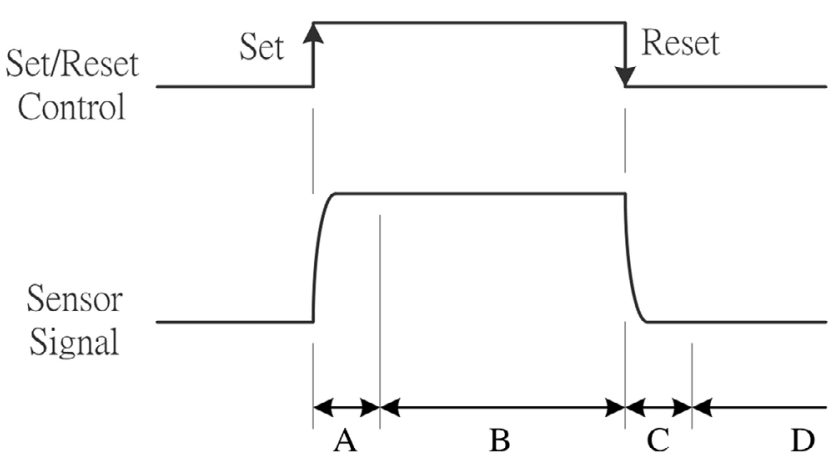

Fig. 3. Four phases of control are timed through one cycle of measurement: (A) settling time after the switch to Set-state, (B) signal in Set-state is ready for measuring, (C) settling time after the switch to Reset-state, (D) signal in Reset-state is ready for measuring.

"Set-state" data sampling phases (phase A and B), and "Reset-state" switching and "Reset-state" data sampling phases (phase $\mathrm{C}$ and $\mathrm{D}$ ). Because the sensor signal is flipping at the moment of "Set/Reset" switching, a short period of settling time (phases $\mathrm{A}$ and $\mathrm{C}$ ) is required before the data is acquired.

\section{PRE-FLIGHT CALIBRATION}

As illustrated in Fig. 4, there are various sources that can introduce errors to the measurement values of MRM. These include an inherent cross-axis effect (Kubík et al. 2006; Včelák et al. 2006), a bridge offset of the sensor, circuit offset of signal amplification and acquirement, residual magnetic field of module board and chassis (so called module-level local bias, MLB), misalignment and gain scaling factor of non-identical sensor axes, and a temperature dependent gain scaling factor. These errors must be calibrated before the magnetometer is installed in the satellite. In this section, we briefly describe the errors involved on the way of generating instrumental signals. Since the magnetic field data will be derived from these output signals, we propose a calibration method to compensate for these errors in the raw data provided by an AMR measuring system.

\subsection{Mathematical Model}

The process of converting an ambient magnetic field vector $B=\left[\begin{array}{lll}b_{1} & b_{2} & b_{3}\end{array}\right]$ in an orthogonal frame system (OFS) into tri-axial signals $S=\left[s_{1} s_{2} s_{3}\right]$ in an instrument reference frame system (IRFS) under AMR sensors' Set-state and Reset-state can be separately modelled as follows (Caruso 2003):

$S_{S E T}=\left(B+B_{M L B}\right) T_{S E T}+\left(B+B_{M L B}\right) T_{C A}+D$

$S_{\text {RESET }}=\left(B+B_{M L B}\right) T_{R E S E T}+\left(B+B_{M L B}\right) T_{C A}+D$

The transform matrix $T_{S E T}=-T_{\text {RESET }}=T_{S}=\left[\begin{array}{lll}t_{11} & t_{12} & t_{13} \\ t_{21} & t_{22} & t_{23} \\ t_{31} & t_{32} & t_{33}\end{array}\right]$ contains information of misalignments and gains scaling factors when transforming magnetic field from OFS to IRFS. The other matrix $T_{C A}=\left[\begin{array}{ccc}0 & c_{12} & c_{13} \\ c_{21} & 0 & c_{23} \\ c_{31} & c_{32} & 0\end{array}\right]$ represents the offset resulting from the cross-axis effect. Vector $B_{\mathrm{MLB}}=\left[\begin{array}{ll}v_{1} & v_{2} \\ v_{3}\end{array}\right]$ is the module-level local bias, and vector $D=\left[d_{1} d_{2} d_{3}\right]$ is a combination of the circuit offset and bridge offset. Applying the "Set/Reset" mechanism as mentioned in section 2.3, we can eliminate the deviations of measurements due to cross-axis effect, bridge offset and circuit offset by subtracting Eq. (2) from Eq. (1), and obtain

$S_{S E T}-S_{\text {RESET }}=\left(B+B_{M L B}\right)\left(T_{S E T}-T_{R E S E T}\right)=2\left(B+B_{M L B}\right) T_{S}$

The original magnetic field vector $B$ can be derived from raw data by re-arranging Eq. (3) into the measurement model: 


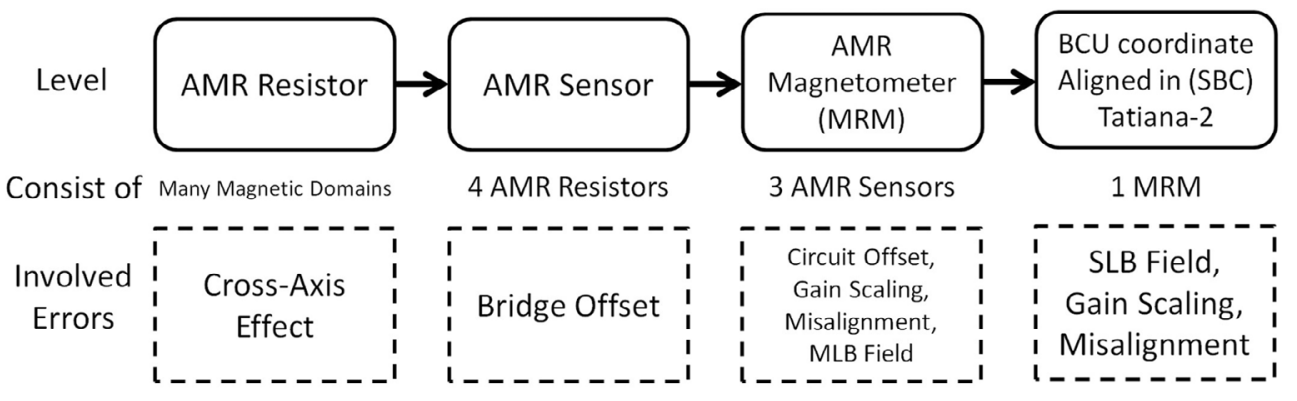

Fig. 4. AMR magnetometer construction and involved errors to be compensated.

$B=0.5\left(S_{S E T}-S_{R E S E T}\right) T_{S}^{-1}-B_{M L B}=m A+u$

In Eq. (4), $A=0.5 T_{S}^{-1}=\left[\begin{array}{lll}a_{11} & a_{12} & a_{13} \\ a_{21} & a_{22} & a_{23} \\ a_{31} & a_{32} & a_{33}\end{array}\right], m=S_{\text {SET }}-S_{\text {RESET }}, u=$ $-B_{M L B}$.

The transform matrix $A$ contains information with regard to misalignments and gains scaling factors when reconstructing magnetic field $B$ from IRFS to OFS. The vector $u$ is a modifier of module-level local bias (MLB). For each magnetometer, its parameters of matrix $A$ and vector $u$ will be calibrated by known magnetic test fields according to the procedure described in the next section.

\subsection{Calibration Method}

In the calibration process, we need to find a set of parameters for the measurement model [Eq. (4)] whose estimates of the test fields match the known test settings the best. Then, the calibrated measurement model may be applied to derive the measurement of an unknown field inflight.

We apply a set of magnetic test fields $\left\{B_{i}, i=1, \ldots, \mathrm{n}\right\}$ to the MRM under calibration to obtain its corresponding signal data $\left\{m_{i}\right\}$. The best set of parameters which would estimate $B$ based on $m$ over the full dynamic range would be the one minimizing the sum $R$ of the squares of the estimation mismatches as shown in Eq. (5).

$R=\sum_{i=1}^{n}\left[B_{i}-\left(u+m_{i} A\right)\right]^{2}$

Since the measurement model in Eq. (4) is linear (in parameters), the best parameter set can be obtained by solving the multi linear regression fit with the following formula,

$\underset{4 \times 3}{\Phi}=\left[\begin{array}{l}u \\ A\end{array}\right]=\left(\underset{4 \times n}{X_{n \times 4}^{T}} \underset{n \times 4}{X}\right)^{-1} \underset{4 \times n}{X} \underset{n \times 3}{Y}$ where $X=\left[\begin{array}{cc}1 & m_{1} \\ 1 & m_{2} \\ \vdots & \vdots \\ 1 & m_{n}\end{array}\right]=\left[\begin{array}{cc}1 & S_{S E T(1)}-S_{\text {RESET(1) }} \\ 1 & S_{S E T(2)}-S_{\text {RESET(2) }} \\ \vdots & \vdots \\ 1 & S_{S E T(n)}-S_{\text {RESET(n) }}\end{array}\right], Y=\left[\begin{array}{c}B_{1} \\ B_{2} \\ \vdots \\ B_{n}\end{array}\right]$

To calibrate, we first use a magnetic chamber to generate a test field set $\left\{B_{i}\right\}$ as a measurement reference set and record their corresponding measurement data pairs $\left\{S_{S E T(i)}\right.$, $\left.S_{\text {RESET }(i)}\right\}$. Then, we organize these references and measurement data into matrices $Y$ and $X$ in Eq. (6) to obtain the calibration parameters in matrix $A$ and vector $u$ from the solution matrix $\Phi$. To obtain a good estimation on $A$ and $u$, the set of test field values, $\left\{B_{i}\right\}$ should cover the full dynamic range of the sensor measurement with independent axes variations as shown in the first panel of Figs. 5 and 6. This way, the variation content of $\underset{n \times 4}{X}$ is rich and its cross covariance matrix $\underset{4 \times n}{X} \underset{n \times 4}{X}$ would not become ill conditioned.

\subsection{Results of Calibration}

Figure 5 shows the raw data of the MRM subjected to stepwise magnetic field tests along each axis. The sensor temperature was about $40^{\circ} \mathrm{C}$ during the experiment. The result of calibration is shown in the second panel of Fig. 6, the bridge offset, cross-axis effect, circuit offset, modulelevel local bias (MLB), misalignment and gain scaling factor were all properly calibrated. The calibration parameters of the flight unit are shown below.

$$
A=\left[\begin{array}{lll}
-1.8477 & +0.0240 & -0.1145 \\
-0.0294 & -1.9008 & +0.0248 \\
-0.0676 & -0.0149 & +1.8770
\end{array}\right], u=-B_{M L B}=\left[\begin{array}{c}
-877 \mathrm{nT} \\
-426 \mathrm{nT} \\
+493 \mathrm{nT}
\end{array}\right]^{T}
$$

The correlation coefficients of the fit (Abdi 2007) for the three axes are 1 subtracted by the ratio of the variance of residual errors to the total variance of their test field. These ratios along the $\mathrm{x}, \mathrm{y}, \mathrm{z}$ axes are $0.000161,0.000155$, and 0.000162 respectively. 


\section{IN-FLIGHT DATA ANALYSIS}

Samples of fully calibrated MRM data are analyzed to examine the geomagnetic field structure at a global scale and field-aligned current pattern on a scale of a few hundreds $\mathrm{km}$. As discussed in the following paragraphs, the in-flight data of MRM did confirm that the NCU built magnetometer is reliable and is a qualified instrument for future space missions.

\subsection{In-Flight Calibration}

The Technical Master (TM) magnetometer on the Tatiana-2 is a calibrated vector magnetometer under the satellite bus coordinates (SBC). Although the available downlink rate of TM data is only one vector per minute, it's sufficient to be a reference of in-flight calibration of the MRM. Since there are orientation differences and also scaling factor modification and offsets caused by the satellite body when
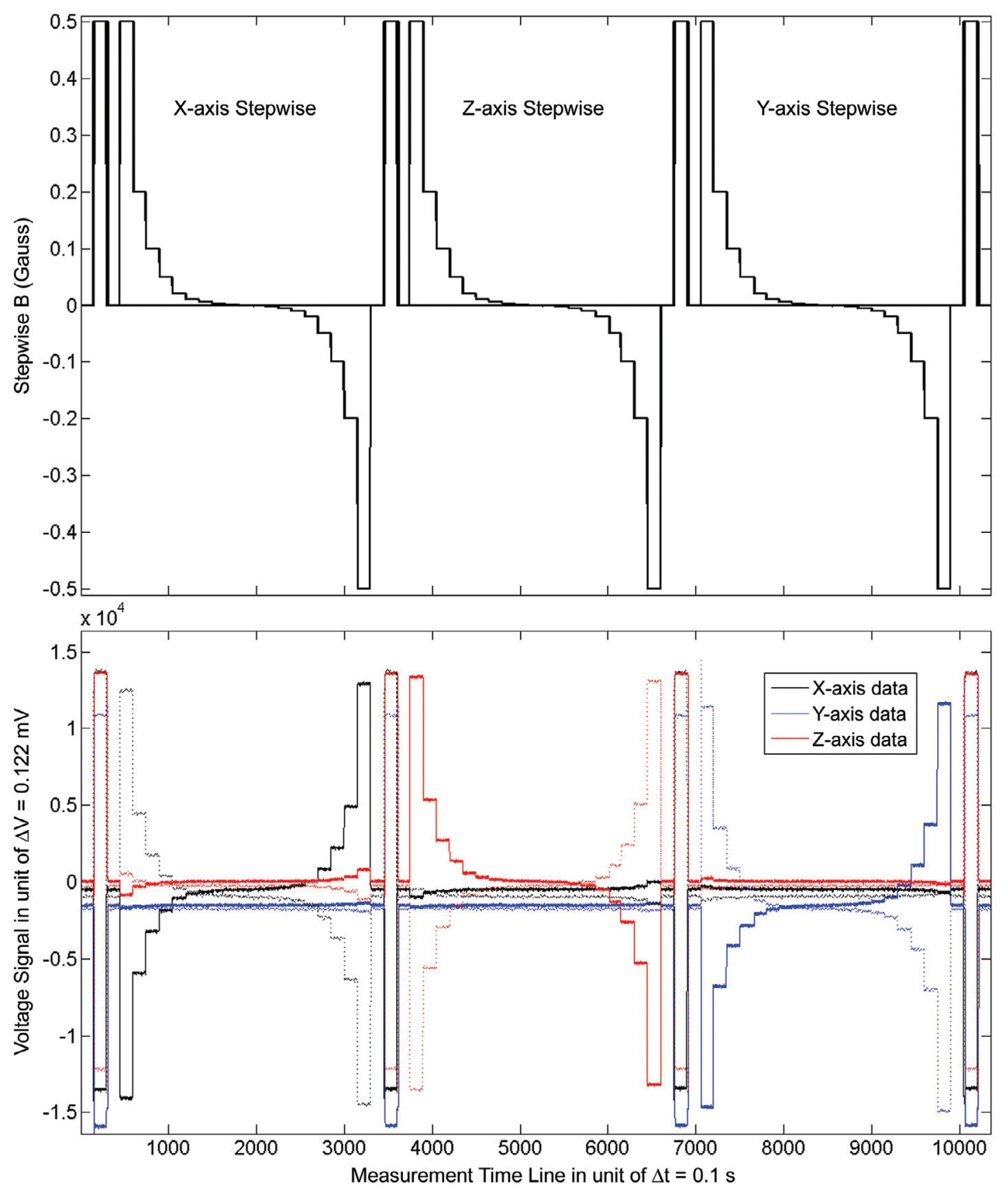

Fig. 5. Stepwise magnetic test field is shown in the first panel and the corresponding raw data of MRM are shown in the second panel where the solid and dotted lines correspond to data in Set and Reset states respectively. The magnetic field in each axis is exerted along $\mathrm{x}, \mathrm{z}, \mathrm{y}$ axes of the OFS independently in time durations of 400 to 3400,3700 to 6700 , and 7000 to 10000 respectively. There are 19 test steps along each axis. Obviously, there exist offsets, unbalanced gains and misalignments among axes in the raw data. 


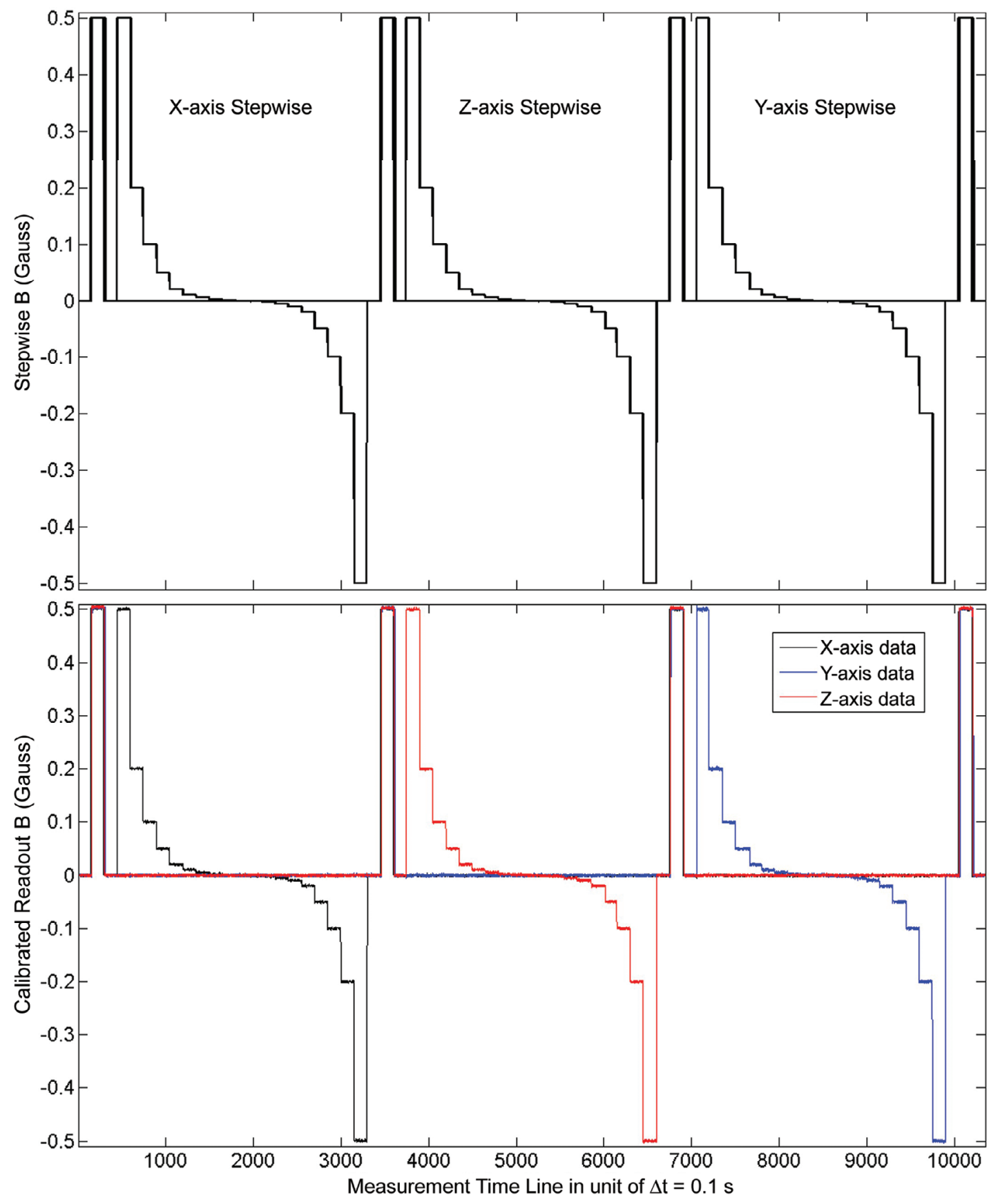

Fig. 6. After pre-flight calibration, the calibrated MRM measurement is shown in the second panel, which matches very well the test field set by the Helmholtz chamber. The STD of the linearity error over the range of -0.5 to +0.5 Gauss along the $\mathrm{x}, \mathrm{y}$ and $\mathrm{z}$ axes are 25,26 , and $23 \mathrm{nT}$ respectively.

MRM was installed onto the satellite, the relation between TM data $B_{T M}$ and MRM data $B_{M R M}$ can be expressed as the following equation:

$$
B_{T M}=\left(B_{M R M}-B_{S L B}\right) T_{R}+\varepsilon_{1}
$$

Here $B_{S L B}$ is the satellite-caused local bias (SLB), $T_{R}$ is the rotation matrix with respect to $\mathrm{TM}$, while $\varepsilon_{1}$ is the residual variation of the in-flight calibration. Notice that: $\varepsilon_{1}$ includes the variance and the calibration residuals of the TM magnetometer which should have a much senior space flight heritage. Applying the least-square method to calculate the parameters:

$$
T_{R}=\left[\begin{array}{rrr}
-0.0173 & 1.2190 & 0.0317 \\
1.0584 & -0.0288 & -0.1395 \\
-0.0009 & 0.0284 & 1.1248
\end{array}\right], B_{\text {SLL }}=\left[\begin{array}{r}
213 \mathrm{nT} \\
-2196 \mathrm{nT} \\
14577 \mathrm{nT}
\end{array}\right]^{T}
$$


Figure 7 shows three types of data lines on 18 January 2010, of MRM data, TM data and MRM data calibrated by TM data in the SBC as the geomagnetic fields were measured. There exists a large satellite-caused local bias in the $\mathrm{z}$-axis of MRM (in ram direction of satellite). Otherwise, it could be concluded that MRM data are in good consistence with the TM data. Since MRM benefits from the thermal insulation blanket, the shadowing of the solar panel, and the heat conduction contact with the satellite, the sensors are kept at a stable temperature around $40^{\circ} \mathrm{C}$ during operation as shown in the last panel of the figure. Hence we needn't put too much effort to calibrate temperature drift.

\subsection{Trending Verification of MRM In-Flight Measurement}

The validity of MRM in-flight data can be verified by checking the consistency between its trending with the dominant features of the geomagnetic field. The strength of the vertical magnetic component is the strongest when the satellite flies cross polar areas (downward in the northern polar region and upward in the southern polar region) while it is the weakest when satellite passes through the equator. The vertical data component shown in red in Fig. 8 reveals this feature vividly. The validation of the MRM in-flight data with the IGRF model (WDC for Geomag 2010) is also provided in a companion paper by Jiang et al. (2012).

\subsection{Feature of Field Aligned Current}

The fully calibrated MRM data are analyzed to examine the magnetic field disturbances generated by field aligned currents. Figure 9 presents a case of simultaneous observations of the magnetic field and floating potential, respectively, by the MRM and ETP of the BCU payload on a geomagneticly quiet day of 18 January 2010 . The MRM data were detrended first by removing their base line obtained by 160 data point $(\sim 500 \mathrm{~km})$ smoothing. The detrended MRM data are shown in the second panel of Fig. 9; there are apparent out of ordinary variations in $\Delta B_{\text {east }}$ and $\Delta B_{\text {north }}$ when the satellite travelled north bound in the dark

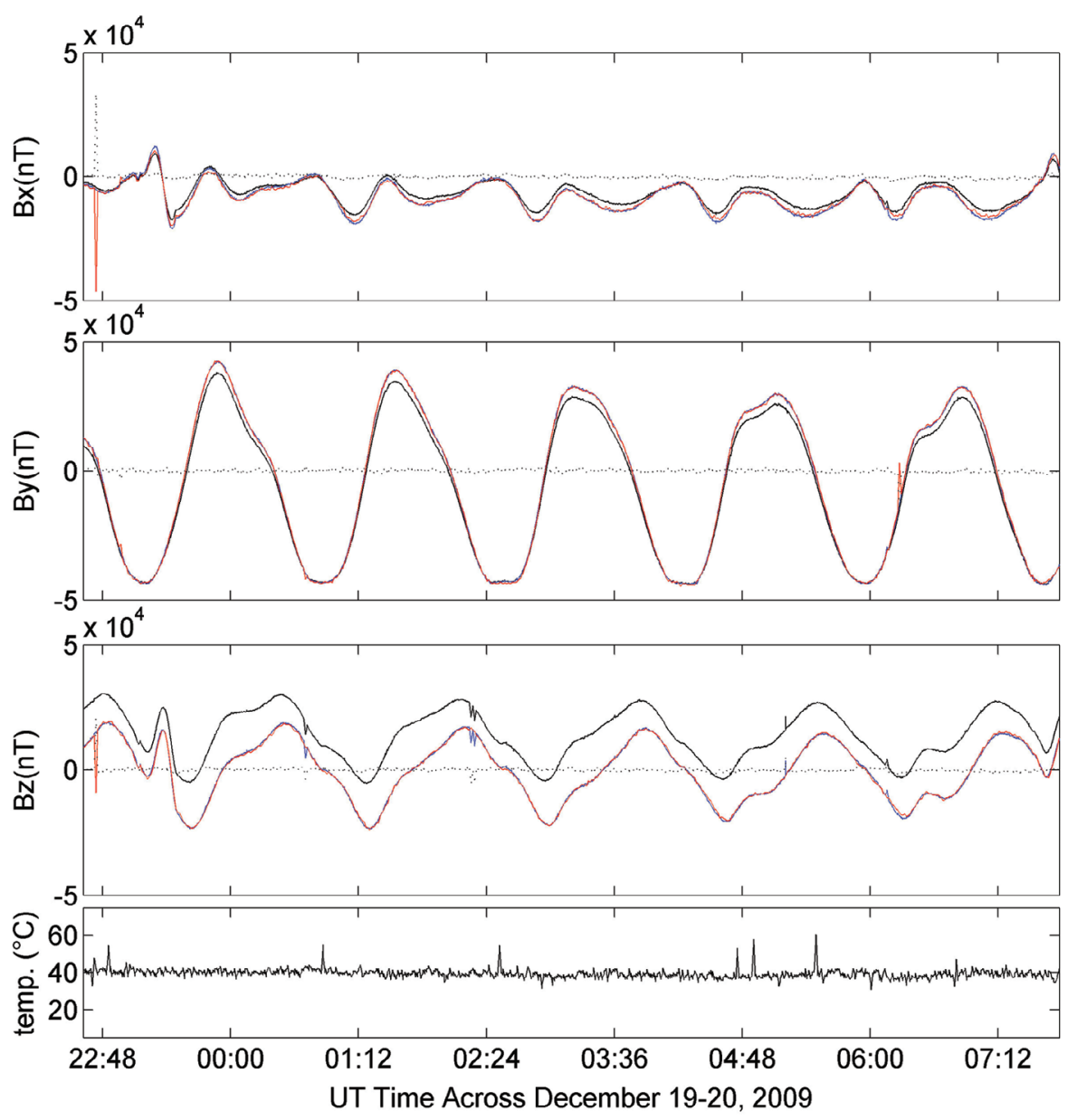

Fig. 7. The match between the MRM data (black line), Tatiana-2 TM data (red line) and MRM data calibrated by Tatiana-2 TM data with satellitelevel local bias removed (blue line). The top panel is Bx: the horizontal right component, the second panel By: the nadir direction, and the third panel Bz: the ram direction. The dotted lines around $0 \mathrm{nT}$ in the first, second and third panels are the calibration residuals of MRM by TM. The in-flight sensor temperature is rather stable as shown in the last panel. 

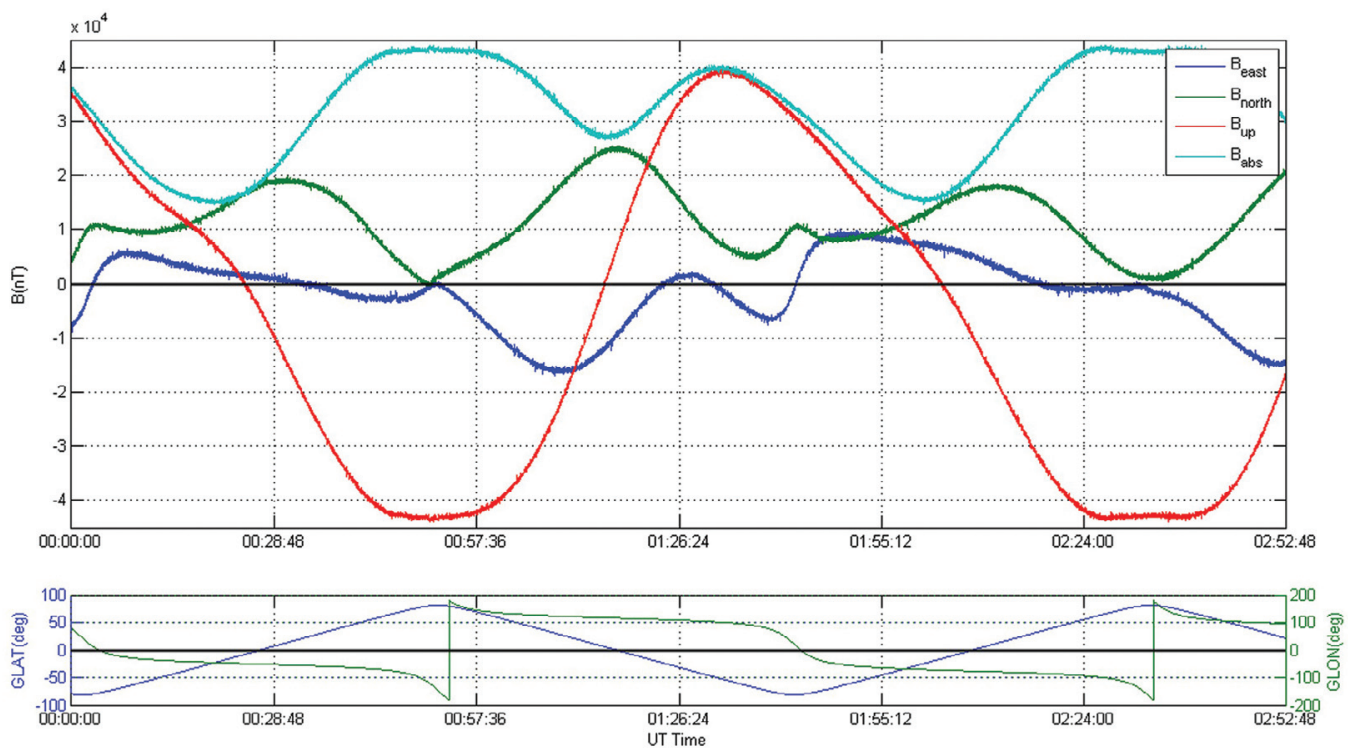

Fig. 8. The MRM in-flight data components in geographic East-North-Upward coordinate (ENU) and their corresponding orbital information, which are generated by "TrakStar" (Kelso 2007) according to the time tags of in-flight data.

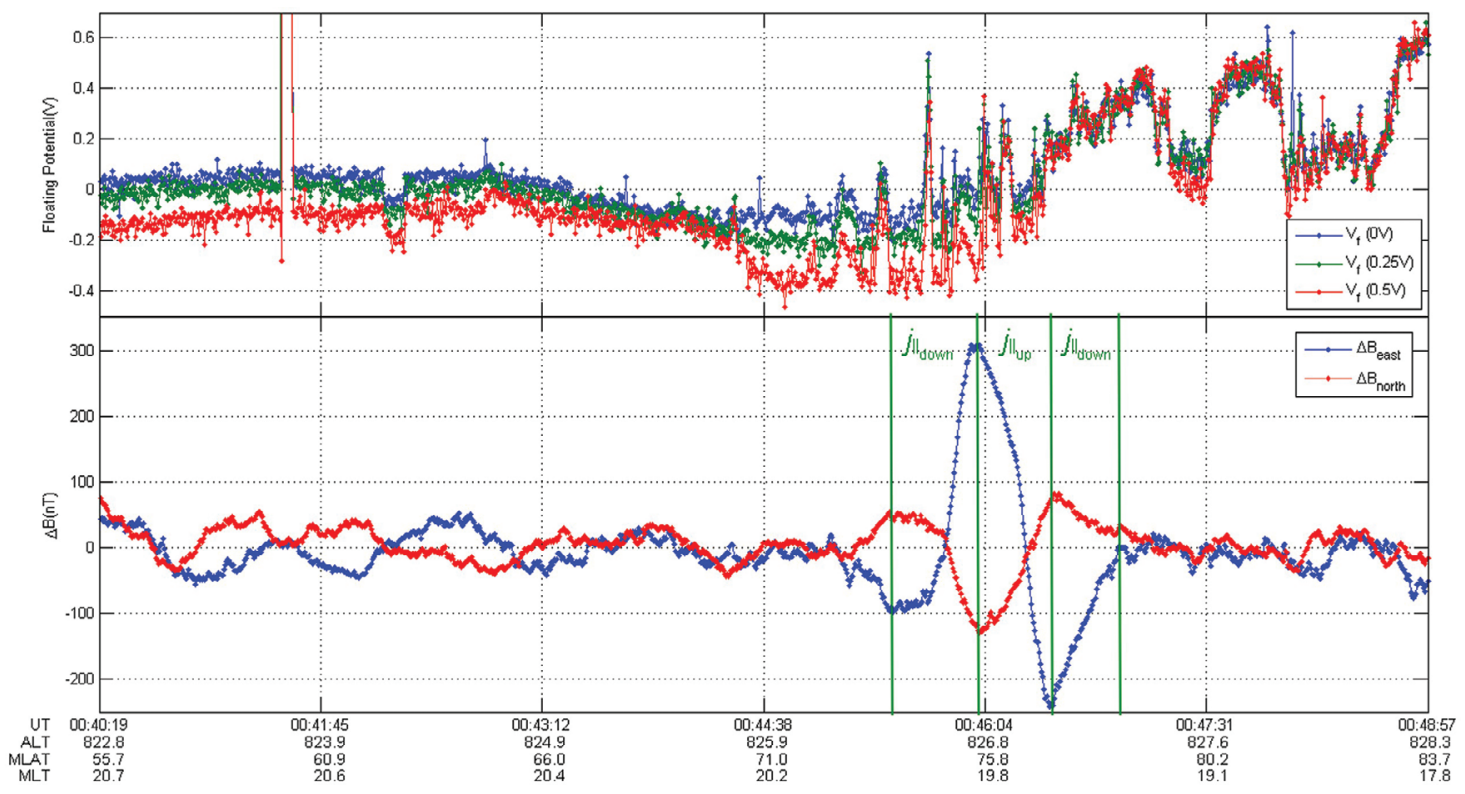

Fig. 9. Detrended MRM waveform featuring the field-aligned current.

around 20 to 19 magnetic local time (MLT) from about $74^{\circ}$ magnetic latitude (MLAT) to $77^{\circ}$ MLAT through the nominal auroral region. The corresponding field-aligned current (FAC) density can be calculated by the Ampere's law (In the ionosphere the displacement current can be discounted): $\vec{J}=\frac{1}{\mu_{0}}(\nabla \times \vec{B})$.

At high latitude, the geomagnetic field direction is nearly parallel (or anti-parallel) to the geographic vertical direction, thus field aligned current density $\left(J_{\| I}\right)$ can be derived from the horizontal $\vec{B}$ field components along the satellite path as:

$J_{/ /} \cong \frac{1}{\mu_{0}}\left(\frac{\partial \Delta B_{\text {north }}}{\partial D_{\text {east }}}-\frac{\partial \Delta B_{\text {east }}}{\partial D_{\text {north }}}\right)$

Based upon Eq. (10), we can identify the downward FAC in the region where $\Delta B_{\text {east }}$ increases with increasing northward distance (i.e., positive slope) and $\Delta B_{\text {north }}$ decreases with increasing eastward distance (i.e., negative slope), while the upward FAC in the region where the signs of the 
corresponding differential terms are reversed. These current sheets are depicted in the bottom panel of Fig. 9. As shown in the panel, the steepest slopes were found at the latitudes adjacent to $75^{\circ}$ MLAT with upward field aligned current located at higher latitudes.

Simultaneous floating potential $\left(\mathrm{V}_{\mathrm{f}}\right)$ measurements by ETP under the three applied sine wave voltages $(0,0.25$, $0.5 \mathrm{~V}$ ) are plotted at the top panel of Fig. 9 for comparison. It is noticeable in the figure that highly fluctuating $\mathrm{V}_{\mathrm{f}}$ patterns are observed in the regions co-located with the FAC distributions. Previous observations have suggested that high energy charged particles of magnetospheric origin can play a dominant role in the current balance to the spacecraft (or the surface of a probe) in the dark high-latitude ionosphere (e.g., Yeh and Gussenhoven 1987). Since the floating potential is determined on the basis of the current balance condition at ETP, fluctuating floating potentials are expected in the environment of thermal plasma mixing with energetic particles that carry field aligned currents. Thus the ETP observations provide further evidence of the existence of field aligned currents in the regions identified from the MRM measurements.

By means of Eq. (10), we further calculate the intensities of these field aligned currents. The result indicates that the maxima current density is about 2 and $3 \mu \mathrm{A} \mathrm{m} \mathrm{m}^{-2}$, respectively, for downward and upward currents. These magnitudes are within the typical range of FAC observed by other satellites (e.g., Iijima and Potemra 1982; Elphic et al. 2001).

\section{CONCLUSIONS}

With properly designed control sequence and the implementation of a "Set/Reset" magnetizing procedure to compensate the offset of the sensor, the MRM is able to provide good quality data. Even though the MRM on Tatiana-2 is subjected to a magnetic bias of 0.146 Gauss, its measurement can be successfully calibrated to that of the main TM magnetometer of Tatiana-2. Despite the MRM was sampled at only $2.2 \mathrm{~Hz}$ due to a limited downlink budget for the BCU, the phenomenon of field-aligned current at high latitudes is observed by the detection of the magnetic field disturbances perpendicular to the current. The measured current density is about $2 \sim 3 \mu \mathrm{A} \mathrm{m}^{-2}$. It proves that the MRM and its supporting payload system are space qualified to survive at least 4 months for the detection of magnetic field variations with expected magnitude and spatial extent typically induced by the field-aligned currents.

On the basis of the successful observation of the fieldaligned current, we have more confidence in optimizing the performance of the MRM to detect the space weather events characterized by smaller and/or faster magnetic field changes. In addition, to improve the MRM for a higher sampling rate and resolution, there are other desirable issues for improvement, such as a smaller size and lighter weight. We also plan to separate the size-reduced AMR sensor head from the main electrical circuits for remote deployment so the further sensitized sensor head can be free from minute disturbances caused by the magnetic fields generated inside the payload and other satellite subsystems.

Acknowledgements We are indebted to continual project grants from the "National Science Council" and "National Space Organization (NSPO)," e.g., 94-NSPO(A)-PC-FA0701, 95-NSPO(B)-SE-FA07-02, 97-NSPO(B)-SE-FA07-02, 98-NSPO(A)-GE-F07-02, etc. We would like to thank Russian Colleagues for their support on the preparation and installation of BCU payload onto the satellite. We are also greatly indebted to Professor Koh-Ichiro Oyama for his passionate encouragement and guidance in the ETP technology.

\section{REFERENCES}

Abdi, H., 2007: Multiple correlation coefficient. In: Salkind, N. J. (Ed.), Encyclopedia of Measurement and Statistics, Thousand Oaks (CA): Sage Publications, Inc., 648-651.

Caruso, M. J., 2003: Cross axis effect for AMR magnetic sensors. Application Note AN215, Honeywell International, Inc.

Caruso, M. J., T. Bratland, C. H. Smith, and R. Schneider, 1999: Anisotropic magnetoresistive sensors: Theory and applications. Sensors, 16.

Caruso, M. J., T. Bratland, C. H. Smith, and R. Schneider, 1998: A new perspective on magnetic field sensing. Sensors Expo Proceedings, October 1998, 195-213.

Chou, C. J., 2008: Compensable high resolution three dimensional magnetometer. Master Thesis, Institute of Opto-Mechatronics Engineering, National Central University, Jongli, Taiwan.

Elphic, R. C., J. D. Means, R. C. Snare, R. J. Strangeway, L. Kepko, and R. E. Ergun, 2001: Magnetic field instruments for the FAST auroral snapshot explorer. Space Sci. Rev., 98, 151-168, doi: 10.1023/A:10131536233 44. [Link]

Iijima, T. and T. A. Potemra, 1982: The relationship between interplanetary quantities and Birkeland current densities. Geophys. Res. Lett., 9, 442-445, doi: 10.1029/GL 009i004p00442. [Link]

Jiang, S. B., T. L. Yeh, H. C. Yeh, J. Y. Liu, Y. H. Hsu, and L. Y. Liu, 2012: System Architecture of the BCU payload on Tatiana-2. Terr. Atmos. Ocean. Sci., 23, 193208, doi: 10.3319/TAO.2011.09.20.01(AA). [Link]

Kelso, T. S., 2007: "TrakStar" version 2.65, the Satellite Tracking Software, available at http://celestrak.com/ software/tskelso-sw.asp.

Kubík, J., J. Včelák, and P. Ripka, 2006: On cross-axis ef- 
fect of the anisotropic magnetoresistive sensors. Sens. Actuators A: Phys., 129, 15-19, doi: 10.1016/j.sna.20 05.09.053. [Link]

Liu, L. Y., 2005: Research and development of magnetometer. Master Thesis, Institute of Opto-Mechatronics Engineering, National Central University, Jongli, Taiwan.

Pant, B. B. and M. J. Caruso, 1996: Magnetic sensor crossaxis effect. Application Note AN 205, Honeywell International, Inc.

Včelák, J., P. Ripka, A. Platil, J. Kubík, and P. Kašpar, 2006: Errors of AMR compass and methods of their compensation. Sens. Actuators A: Phys., 129, 53-57, doi: 10.1016/j.sna.2005.09.048. [Link]

WDC for Geomagnetism, 2010: International Geomagnetic
Reference Field, availavle at http://wdc.kugi.kyoto-u. ac.jp/index.html.

Yang, B. R., 2002: Measuring and research of the magnetic field structure generated by the current-driven precision linear encoder. Master Thesis, Institute of Mechanical Engineering, National Central University, Jongli, Taiwan.

Yang, J. J., 1997: Fabrication and characterization of nickeldeposited magnetic thin-film. Master Thesis, Institute of Mechanical Engineering, National Central University, Jongli, Taiwan.

Yeh, H. C. and M. S. Gussenhoven, 1987: The statistical electron environment for Defense Meteorological Satellite Program eclipse charging. J. Geophys. Res., 92, 7705-7715, doi: 10.1029/JA092iA07p07705. [Link] 